\title{
Chemotherapy with consideration to biological rhythm reduce oxaliplatin- induced peripheral neuropathy
}

\section{Yoshihiro Seto, Masashi Wanifuchi, Aya Takahara, Shunsuke Takakura, Fumiyasu Okazaki, Mari Tomonari, Hideto To}

Department of Medical Pharmaceutics, Graduate School of Medicine and Pharmaceutical Sciences for Research, University of Toyama, Toyama, Japan

\section{Background}

Although oxaliplatin (L-OHP), which is the third platinum anti-cancer drug, plays an important role in treatment of colorectal cancer, cold hyperalgesia is caused to patients used L-OHP, and effective methods of prevention or amelioration against the adverse effect are not yet established. In the past study, we showed that cisplatin-induced peripheral neuropathy was ameliorated by the selection of optimal dosing time in rats. In the present study, we investigated the influence of dosing times on L-OHP-induced cold hyperalgesia in mice.

\section{Methods}

L-OHP (5 mg/kg), Pt(dach)Cl2 (4.8 mg/kg), or oxalate $(1.7 \mathrm{mg} / \mathrm{kg})$ was administered intravenously at 1:00, 5:00, 9:00, 13:00, 17:00, and 21:00 to male C57BL/6J mice, and vehicle was given to the control group. To assess the cold hyperalgesia, acetone test was performed in assessment days. To reveal the relation between oxaliplatin-induced cold hyperalgesia and transient receptor potential ankyrin 1 (TRPA1) and melastatin 8 (TRPM8), each antagonist was treated 30 minutes before the acetone test.

3. Results

The L-OHP 1:00, 17:00, and 21:00-treated groups did not develop the withdrawal responses 2 hours after the administration. The other L-OHP-treated groups, especially 9:00-treated group, significantly increased the number of withdrawal responses compared with the control group. After a single administration, the 9:00-treated group only showed significantly higher withdrawal responses than the control group in all measured points.

L-OHP is metabolized to the 2 compounds, $\mathrm{Pt}(\mathrm{dach}) \mathrm{Cl} 2$ and oxalate. There were no significant dosing-time dependent differences in response to cold stimulation in $\mathrm{Pt}(\mathrm{dach}) \mathrm{Cl} 2$-treated groups. On the other hand, the 9:00-treated group showed the highest responses to cold stimulation among all groups when the oxalate was given.

Furthermore, TRPA1 antagonist reduced cold hyperalgesia 2 hours and did not inhibit the response after the administration.TRPM8 antagonist did not reduce cold hyperalgesia 2 hours and inhibited cold hyperalgesia 3 days after the administration.

\section{Conclusions}

It was revealed that L-OHP-induced cold hyperalgesia was most severe in the 9:00-treated group in the all dosing groups. Furthermore, these dosing time-dependent differences were related to oxalate which is one of the metabolized compound of L-OHP. 